\section{MicroRNA from dying neurons triggers astrocytosis in ALS}

Motor neurons that die in amyotrophic lateral sclerosis (ALS) release microRNAs (miRNAs) that provoke an aberrant reactive response in astrocytes, according to a new study. Astrocytic uptake of miR-218 from the extracellular environment caused changes in protein expression that were associated with ALS and astrogliosis. These changes could be reversed using an antisense oligonucleotide (ASO), suggesting that this process can be targeted therapeutically.

Pathological aggregates in neurodegenerative diseases such as ALS primarily accumulate within neurons, where they are thought to lead to cell death. However, dysfunctional glial reactivity also plays an important part in disease progression and can accelerate neuronal loss. The molecular mechanisms underlying this aberrant glial activity are poorly understood.
Previously, Mariah Hoye and colleagues found that miR-218 was highly prevalent in motor extracellular space after neuronal death in ALS model rats. "This was very surprising and exciting," remarks Hoye. "We wondered whether miRNAs released from dying motor neurons could signal to neighbouring glia and cause them to become reactive."

In the new study, the team showed that cultured astrocytes take up extracellular miR-218 released from lysed motor neurons. Interestingly, miR-218 bound to transcripts of EAAT2, an astrocytic glutamate transporter previously shown to be depleted in ALS, and supressed its expression. This repression could be reversed with an ASO targeted to miR-218.

The team found that anti-miR-218 ASOs also rescued EAAT2 expression neurons, and was released into the

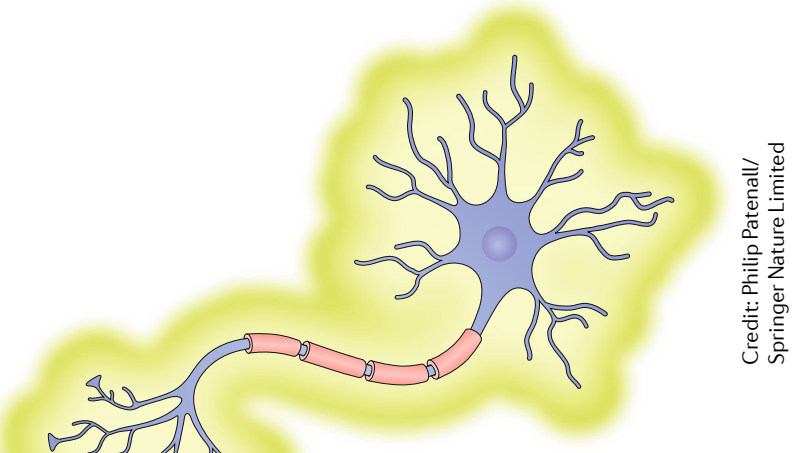

in a live mouse model of ALS.

Furthermore, these ASOs reversed upregulation of the astrogliosis markers GFAP and Cx43 in these animals.

"While many neurodegenerative diseases start in neurons, we now know that glia are the most substantial contributors to disease progression, so modulating their response to neuronal injury could be very therapeutically promising," comments Hoye. The team now we now know that glia are the most substantial contributors to disease progression

5

\section{Artificial intelligence accelerates detection of neurological illness}

A new deep-learning-based artificial intelligence system can identify a variety of acute neurological disorders in patient $\mathrm{CT}$ scans within seconds. The tool might enable neurologists to rapidly identify patients who need urgent attention and prioritize diagnosis and treatment in these individuals.

Swift diagnosis and treatment of acute neurological diseases such as stroke is crucial to minimize progressive neurological damage. Computerized analysis of patient brain images has the potential to help clinicians to diagnose and treat patients more rapidly than by human judgement alone.

"I was motivated by my experiences of taking care of patients with acute neurological illnesses, in which any possible way of reducing the time it took for us to reach these individuals could have improved their outcomes," explains corresponding author Eric
Oermann. "One day, Joseph Titano and I said to ourselves that we needed to solve this problem with deep learning."

The researchers used a database of over 37,000 head CT scans featuring a variety of neurological findings. An artificial intelligence system known as a $3 \mathrm{D}$ convolutional neural network was trained to identify whether or not scans contained "critical findings" signifying the presence of acute neurological disease such as stroke, haemorrhage or hydrocephalus.

Compared with human judgement, the computerized system could correctly detect critical neurological findings with the same sensitivity (79\%) but a lower specificity ( $48 \%$ versus $85 \%)$. From these results, Oermann and colleagues predicted that their system could be used to prioritize patients who were most likely to require urgent intervention, prompting physicians hope to investigate how extracellular miRNAs released from neurons might affect other types of glia, and whether similar mechanisms might be at work in other neurodegenerative diseases.

Charlotte Ridler

ORIGINAL ARTICLE Hoye, M. L. et al. Motor neuron-derived microRNAs cause astrocyte dysfunction in amyotrophic lateral sclerosis. Brain https://doi.org/10.1093/brain/awy182 (2018)
The endgame is to help patients, and that means translating academic results into actual tools that contribute to patient care to perform rapid diagnosis in these individuals.

To test this idea, the team conducted a randomized controlled trial in a simulated clinical environment to compare the abilities of humans and the automated system to triage brain scans. The automated system processed each image in an average of $1.2 \mathrm{~s}-150$ times more quickly than the human average. Importantly, in the computerprioritized list, scans of individuals requiring urgent attention were assigned a significantly higher queue position than scans of individuals with routine findings.

The team now hope to develop and test their tools in a real clinical setting. "The endgame is to help patients, and that means translating academic results into actual tools that contribute to patient care," comments Oermann.

\section{Charlotte Ridler}

ORIGINAL ARTICLE Titano, J. J. et al. Automated deep-neural-network surveillance of cranial images for acute neurologic events. Nat. Med. https://doi.org/10.1038/s41591-018-0147-y (2018) 